\title{
Residence at Moderate Versus Low Altitude Is Effective at Maintaining Higher Oxygen Saturation During Exercise and Reducing Acute Mountain Sickness Following Fast Ascent to $4559 \mathrm{~m}$
}

\author{
Eva Bernardi, MD; Luca Pomidori, PhD; Davide Cavallari, Dr; Gaia Mandolesi, PhD; Annalisa Cogo, MD, PhD \\ From the University of Ferrara, Department of Biomedical Sciences and Surgical Specialties, Biomedical Sport Studies Center, Ferrara, Italy.
}

\begin{abstract}
Objective.-To continuously monitor oxygen saturation $\left(\mathrm{SpO}_{2}\right)$ by pulse oximeter and assess the development of acute mountain sickness (AMS) using the Lake Louise Score (LLS) during ascent from 1154 to $4559 \mathrm{~m}$ in 2 groups of subjects: 10 moderate-altitude residents (MAR; $\geq 1000-\leq 2500 \mathrm{~m}$ ) and 34 low-altitude residents (LAR). MAR are reported to have a lower incidence of AMS during ascent to higher altitudes compared with LAR. Whether this is related to higher $\mathrm{SpO}_{2}$ is still open to debate.

Methods.-Seventy subjects were recruited; 24-hour $\mathrm{SpO}_{2}$ monitoring with finger pulse oximetry was performed. All subjects rode a cable car from 1154 to $3275 \mathrm{~m}$ and then climbed to $3647 \mathrm{~m}$, where 60 subjects (LAR) overnighted. The second day, 34/60 LAR reached the highest altitude. Ten subjects who lived permanently at 1100 to $1400 \mathrm{~m}$ (MAR) climbed directly to $4559 \mathrm{~m}$ without an overnight stop.

Results. - One LAR was excluded from the analysis because he performed a preacclimatization. We compared data of 10 MAR with data of 33 LAR who reached $4559 \mathrm{~m}$. Two MAR had an LLS of 3, and 8 scored $<3$. Six LAR had an LLS of 3 to 4,8 scored $\geq 5$, and 19 scored $<3$. $\mathrm{SpO}_{2}$ monitoring showed higher mean $\mathrm{SpO}_{2}$ in MAR during ascent above $3600 \mathrm{~m}$ compared with LAR (MAR, 79 $\pm 4 \%$ vs LAR, 76 $\pm 5 \%$; analysis of variance, $P=.03$ ).

Conclusions. - The results of this preliminary study suggest that residence at moderate altitude allows maintenance of higher $\mathrm{SpO}_{2}$ and reduces risk of developing AMS during rapid ascent to higher altitude.
\end{abstract}

Keywords: acclimatization, acute mountain sickness, exercise, oxygen saturation

\section{Introduction}

Progressive exposure to high altitude is characterized by progressive hypoxemia, which worsens with exercise. Travel to elevations above $2500 \mathrm{~m}$ is associated with the risk of developing acute mountain sickness (AMS) or other more severe high-altitude diseases. ${ }^{1}$ AMS is assessed by the Lake Louise Acute Mountain Sickness Scoring System (LLS), a well-validated questionnaire for

Corresponding author: Eva Bernardi, Biomedical Sport Studies Center, Via Gramicia, 3544123 Ferrara, Italy; e-mail: bernardi.eva@ gmail.com.

Submitted for publication May 2016.

Accepted for publication February 2017. field evaluation of AMS. An LLS score $\geq 3$ is diagnostic of altitude sickness. ${ }^{1}$ The best way to prevent AMS is to undertake an adequately slow ascent to high elevation, which allows time for acclimatization. Other nonpharmacologic prophylactic measures for AMS are repeated exposures to hypoxia in the time preceding a high-altitude excursion or spending up to 6 to 7 days at approximately 2200 to $3000 \mathrm{~m}^{2,3}$ In an article regarding the development of AMS at the Regina Margherita Hut, pre-exposure is defined as having spent more than 4 days above $3000 \mathrm{~m}$ during the 2 months preceding the climb.

Likewise, individuals who usually reside at moderate altitude $(\geq 1000-\leq 2500 \mathrm{~m})$ achieve more rapid acclimatization to higher altitude and have reduced 
incidences and severity of AMS compared with those who usually reside at low altitude. ${ }^{5,6}$ The few studies that have addressed this topic reported higher oxygen saturation measured by pulse oximeter $\left(\mathrm{SpO}_{2}\right)$, above $4000 \mathrm{~m}$ in moderate-altitude residents (MAR) compared with low-altitude residents (LAR). ${ }^{5,6}$ In these studies, $\mathrm{SpO}_{2}$ was measured at rest for only a few minutes, and no data during exercise were available. Only 1 study $^{7}$ reported a comparison between endurance performance and $\mathrm{SpO}_{2}$ during exercise in MAR and LAR in a wellcontrolled laboratory setting at $4300 \mathrm{~m}$. This study reported that MAR were able to exercise at a higher intensity and maintained higher exercise $\mathrm{SpO}_{2}$ compared with LAR. However, these results were obtained at the third day of residence at altitude, ${ }^{7}$ when the first changes involved in acclimatization have already occurred, especially the ventilatory acclimatization that contributes to increased $\mathrm{SpO}_{2}$.

No study has continuously monitored $\mathrm{SpO}_{2}$ during progressive high-altitude ascent from moderate to high altitude in MAR compared with LAR, particularly during exercise that induces physiologic responses that may exacerbate the development of AMS.

We had the opportunity to monitor $\mathrm{SpO}_{2}$ and LLS during the entire ascent from Alagna $(1154 \mathrm{~m})$ to the Regina Margherita Hut $(4559 \mathrm{~m})$ in both MAR and LAR. In a previous article, we reported data among LAR. ${ }^{8}$ Now we report data of $\mathrm{SpO}_{2}$ monitoring and LLS in MAR compared with LAR.

\section{Methods}

\section{RECRUITMENT}

We designed a 4-week study on Mt. Rosa at the beginning of the climbing season, aiming to monitor $\mathrm{SpO}_{2}$ for 24 hours and assess the development of AMS in climbers during the ascent to the Regina Margherita Hut $(4559 \mathrm{~m})$. The study purpose was explained in flyers posted in the Alpine Guide office and at the cable car base. Seventy subjects intending to climb to the Regina Margherita Hut were successively enrolled and provided informed consent. All subjects were asked about anthropometric data, altitude of residence, occupation, habitual physical activity, medical history, and previous AMS events. We defined "moderate altitude residents" as subjects who have resided at an altitude between 1000 and $2500 \mathrm{~m}$ for at least 12 months. Subjects taking drugs for AMS prophylaxis were excluded from the study. The study complied with the principles of the Declaration of Helsinki and was approved by the Ethics and Research Committee of the Medical School of the University of Ferrara, Italy.

\section{STUDY DESIGN}

All 70 subjects were equipped with a 24-hour data memory pulse oximeter with a finger sensor (Pulsox300i, Konica Minolta, Osaka, Japan) to monitor $\mathrm{SpO}_{2}$ and heart rate (HR). The pulse oximeter was removed on arrival at the Regina Margherita Hut.

Subjects were asked to complete 2 questionnaires:

1. The Lake Louise Questionnaire for the assessment of AMS while in Alagna, at the Gnifetti Hut (on arrival, in the evening, and on the following morning), and on arrival at the Regina Margherita Hut. On the same sheet, the subjects also recorded the starting and arrival time and any exposure to altitude in the previous 2 months.

2. The Baecke questionnaire ${ }^{9}$ for the measurement of habitual physical activity. This is a self-administered questionnaire separated into 3 dimensions: work, sports, and leisure activity. Each activity is scored in a range from 1 to 5 .

On the first day, subjects rode a cable car from 1154 to $3275 \mathrm{~m}$. They then climbed to the Gnifetti Hut (3647 m), where 60 subjects (all LAR) stayed overnight. On the second day, only 34/60 subjects climbed to the Regina Margherita Hut $(4559 \mathrm{~m})$. Twenty-six subjects ceased further climbing due to bad weather (11), poor health (7), or because the ascent was too demanding (8). Ten of the 70 subjects ( 3 females), permanently residing in Alagna and surrounding area (1100-1400 m, MAR), intended to climb directly from $3275 \mathrm{~m}$ to $4559 \mathrm{~m}$. These subjects were excluded from the previous article. ${ }^{8}$

\section{DATA COLLECTION AND ANALYSIS}

Data were stored at 1-second intervals and processed by DS-5 Minolta software.

For the analysis, we divided the data into 4 frames: at rest at 1154 and $3275 \mathrm{~m}$, during the ascent to the Gnifetti Hut, and during the ascent to the Regina Margherita Hut. The mean values of $\mathrm{HR}, \mathrm{SpO}_{2}$, and time spent under different $\mathrm{SpO}_{2}$ values $(90 \%, 85 \%$, and $80 \%)$ were evaluated. Exercise was identified by the time recorded by the subjects in the information and LLS sheet and confirmed by the HR.

\section{STATISTICAL ANALYSIS}

Statistical analysis was performed using a statistical software package (GraphPad Prism 5; GraphPad Software, San Diego, CA). Data are presented as mean \pm standard deviation where appropriate. Demographic data were analyzed with the unpaired Student's t test. 
Comparison among groups at different altitudes was performed by repeated-measures analysis of variance (mixed model). The Pearson's correlation analysis was used to test the effect of age, body mass index (BMI), and habitual physical activity on LLS score. The level of significance was set at $P<.05$.

\section{Results}

One LAR performed a staged ascent, ${ }^{2}$ spending the previous week at $2300 \mathrm{~m}$ with daily climbing to $3325 \mathrm{~m}$, and was excluded from the analysis. We therefore compared the data of 10 MAR with the data of the remaining $33 \mathrm{LAR}$ who ascended to $4559 \mathrm{~m}$.

No differences were found in age and BMI. Age was $42 \pm 10$ years and $35 \pm 11$ years and BMI was $22.6 \pm 3.3$ $\mathrm{kg} / \mathrm{m}^{2}$ and $22.5 \pm 2.1 \mathrm{~kg} / \mathrm{m}^{2}$ for MAR and LAR, respectively. Two MAR were alpine guides, whereas the others worked in an office. Five LAR had an active job, and 28 worked in an office.

The mean scores of the 3 physical activity items were not significantly different, including physical activity at work (MAR: $2.65 \pm 0.89$; LAR: $2.70 \pm 0.93$ ), sport during leisure time (MAR: 5.86 \pm 2.67 ; LAR: 5.19 \pm 3.3 ), and physical activity excluding sport during leisure time (MAR: $2.87 \pm 0.34$; LAR: $3.16 \pm 0.82$ ). The $P$ values were $.47, .29$, and .07 , respectively.

Regarding pre-exposure to hypoxia, none of the remaining 43 subjects performed a true preacclimatization, according to the definitions reported in the literature $^{2,4}: 2 / 10$ MAR and 5/33 LAR spent 6 to 7 days at $1800 \mathrm{~m}$ hiking up to 2300 to $2500 \mathrm{~m}$ during the previous 2 months. Six additional LAR and one MAR made a 1day excursion above $3500 \mathrm{~m}$ in the previous 2 months.

We considered only the LLS at arrival at $4559 \mathrm{~m}$. Two MAR had an LLS of 3. The remaining 8 had an LLS $<3$. Among the 33 LAR, 6 reported an LLS $\geq 3$ but $<5 ; 8$ reported an LLS $\geq 5$, and the remaining 19 scored $<3$ (Figure). No significant correlation was found between LLS and BMI, age, or physical activity.

We did not find any difference in resting $\mathrm{SpO}_{2}$ at 1154 and $3275 \mathrm{~m}$ nor during exercise from 3275 to $3647 \mathrm{~m}$ (Table). However, MAR maintained a significantly higher $\mathrm{SpO}_{2}$ during exercise from 3647 to $4559 \mathrm{~m}$ (analysis of variance, $P=.004$ ) and spent less time with $\mathrm{SpO}_{2}<90 \%$ (Table).

The mean HR during exercise was not different between MAR and LAR (MAR: $108 \pm 24$ beats/min, LAR: $115 \pm 16$ beats/min).

Regarding the duration of ascent, MAR spent 3 hours and 28 minutes ( \pm 43 minutes) whereas LAR spent 4 hours and 17 minutes ( \pm 1 hour and 15 minutes) to climb from 3647 to $4559 \mathrm{~m}$.

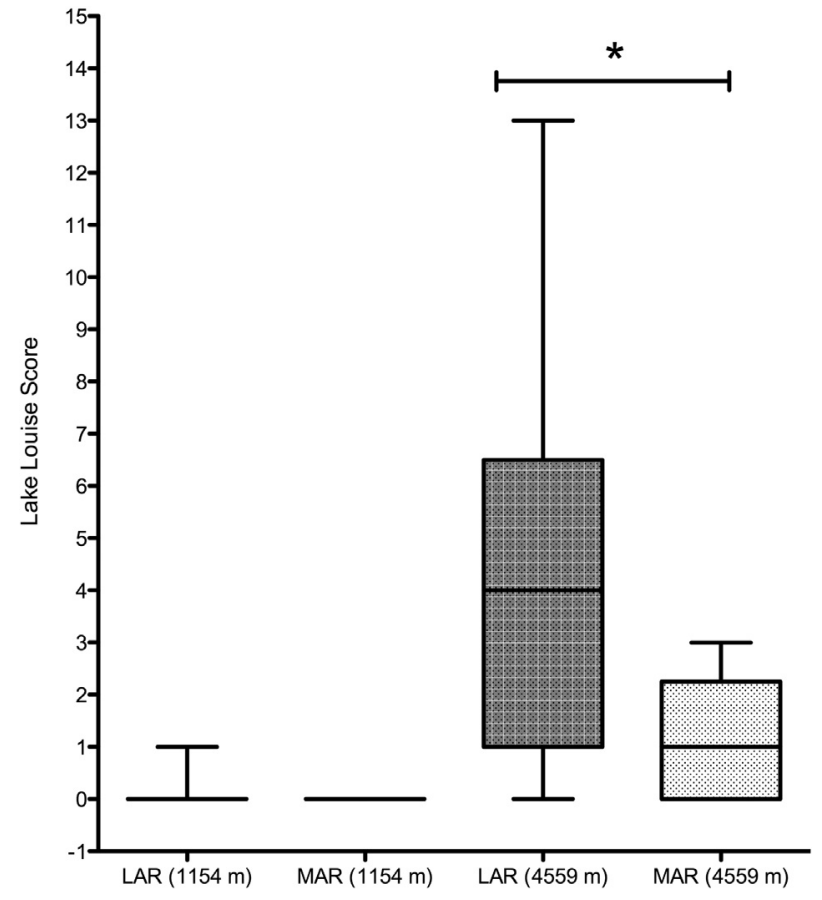

Figure. Lake Louise Score (LLS) in the 2 groups at 1154 and 4559 $\mathrm{m}$. The central box represents the values from the lower to the upper quartiles. The middle line represents the median. The vertical line extends from the minimum to the maximum value. ${ }^{*} P<.05$, Student's $\mathrm{t}$ test. LAR, low altitude residents; MAR, moderate altitude residents.

\section{Discussion}

In this study, we had the opportunity to monitor $\mathrm{SpO}_{2}$ during the entire ascent from Alagna to the Regina Margherita Hut and assess the development of AMS in 2 groups of subjects who permanently resided at low $(<1000 \mathrm{~m})$ or moderate altitude $(\geq 1000-\leq 2500 \mathrm{~m})$. Continuous monitoring of $\mathrm{SpO}_{2}$ allowed us to show that subjects resident at moderate altitude have higher $\mathrm{SpO}_{2}$ during ascent above $3600 \mathrm{~m}$ in comparison with lowlanders. Less severe desaturation is associated with greatly reduced incidence of AMS. The acclimatization acquired while living at moderate altitude presumably allows maintenance of higher $\mathrm{SpO}_{2}$ during the strenuous exertion required to reach higher altitudes, when hypoxic stress is more evident and the need for acclimatization is greater. This peculiarity facilitates tolerance to a hypoxic environment, thus permitting a faster ascent rate than normally recommended. ${ }^{2}$

As reported in the literature, ${ }^{5,6}$ the higher $\mathrm{SpO}_{2}$ in MAR results from ventilatory acclimatization. ${ }^{6}$ A brisk hypoxic ventilatory response is critical during exercise at altitude because it limits the drop in $\mathrm{SpO}_{2}$ and contributes to a left shift of the oxyhemoglobin dissociation curve through induced respiratory alkalosis. Nevertheless, pulmonary limitations affecting $\mathrm{SpO}_{2}$ are still evident in 
people exercising at moderately high altitudes (3000$5000 \mathrm{~m}$ ). In particular, during high-intensity exercise at altitude, hypoxia-induced pulmonary vasoconstriction impairs the ventilation-perfusion ratio, and interstitial pulmonary edema can develop. ${ }^{10}$ Both can worsen $\mathrm{SpO}_{2}$.

Despite the knowledge of the protective effect of residence at moderate compared with low altitude, few studies have focused on the topic. In all but one, $\mathrm{SpO}_{2}$ was measured at rest. In the only article in which $\mathrm{SpO}_{2}$ was measured during exercise, it was measured at the third day of residence at $4300 \mathrm{~m}$ when acclimatization had already started. ${ }^{7}$

We have shown that MAR maintain a significantly higher $\mathrm{SpO}_{2}$ during a fast ascent to $4559 \mathrm{~m}$ and have a low incidence of AMS. In particular, no MAR experienced moderate to severe AMS, whereas 8/33 LAR did experience AMS on arrival at higher altitude. Because we have no data on ventilation, pulmonary artery pressure, or the presence of interstitial pulmonary edema, we cannot know what might be the prevailing mechanism in maintaining better oxygenation during exercise above $3600 \mathrm{~m}$.

Because $\mathrm{SpO}_{2}$ was predominantly different during exercise, we analyzed HR as a marker of exercise intensity between the 2 groups. In fact, if the approach to climb had been slower in MAR, cardiac output would be lower, resulting in less pulmonary diffusion limitation and higher $\mathrm{SpO}_{2}$. According to the available data, we propose that the higher $\mathrm{SpO}_{2}$ observed in MAR is not due to lower exercise intensity. In fact, HR during exercise was similar in the 2 groups.

The mean difference in $\mathrm{SpO}_{2}$ between the 2 groups during ascent to the highest altitude was $3.6 \pm 1 \%$. This represents a lower degree of hypoxic stress. If we consider that the average decline in $\mathrm{SpO}_{2}$ from $1154 \mathrm{~m}$ to $3647 \mathrm{~m}$ amounts to 3.5 to $4 \%$ per $1000 \mathrm{~m}$, then it is as if MAR were at an altitude of at least $800 \mathrm{~m}$ lower, which is an advantage. ${ }^{8}$

\section{Limitations}

The first limitation of this study is the small number of MAR and the variable ascent profile that does not allow the authors to draw firm conclusions. A further limitation is the lack of detailed investigation of physiologic mechanisms influencing $\mathrm{SpO}_{2}$. As such, we cannot identify the reason why differences in $\mathrm{SpO}_{2}$ may exist between MAR and LAR during exercise at high altitude. It should also be noted that the presence of 2 alpine guides among the MAR could have affected the results if they had climbed many times at higher altitudes during the previous weeks. However, this was not the case because we performed the study at the beginning of climbing season (late June to mid-July) and only 1 guide 
had climbed to $4559 \mathrm{~m} 6$ weeks previously without overnight stay. Furthermore, alpine guides can be a selfselected group not only in relation to the hypoxic stressor but also to strenuous exercise and fatigue. However, also most recreational climbers planning the ascent to the Regina Margherita Hut without pharmacological prophylaxis can be a similarly self-selected group. Regarding hypoxic pre-exposure, neither MAR nor LAR followed a true preacclimatization, but both groups included subjects who had already spent some days above $2200 \mathrm{~m}$. This is not unusual for subjects intending to climb Mt. Rosa. Therefore, we do not believe that these differences significantly affected the results.

\section{Conclusion}

This is a preliminary study performed on a small sample of subjects. The results seem to suggest that acclimatization developed during residence at moderate altitude allows maintenance of higher $\mathrm{SpO}_{2}$ during rapid ascent to higher altitude. This reduces the risk of developing AMS. Further research in this area is needed to confirm the results. For example, the study could be repeated with a larger number of subjects, all following the same ascent profile.

Author Contributions: Study concept and design (LP, GM, AC); acquisition of the data (EB, LP, GM); analysis of the data (EB, LP, $\mathrm{GM}, \mathrm{DC}$ ); drafting of the manuscript (EB); critical revision of the manuscript (AC); and approval of final manuscript (AC).

Financial/Material Support: None.

Disclosure Statement: None.

\section{References}

1. Imray $\mathrm{C}$, Booth $\mathrm{A}$, Wright $\mathrm{A}$, Bradwell A. Acute altitude illnesses. BMJ. 2011;343:d4943.
2. Luks AM, McIntosh SE, Grissom CK, et al. Wilderness Medical Society practice guidelines for the prevention and treatment of acute altitude illness: 2014 update. Wilderness Environ Med. 2014;25(4 suppl):S4-S14.

3. Beidleman BA, Fulco CS, Muza SR, et al. Effect of six days of staging on physiologic adjustments and acute mountain sickness during ascent to 4300 meters. High Alt Med Biol. 2009;10:253-260.

4. Schneider M, Bernasch D, Weymann J, Holle R, Bartsch P. Acute mountain sickness: influence of susceptibility, preexposure, and ascent rate. Med Sci Sports Exerc. 2002;34:1886-1891.

5. Staab JE, Beidleman BA, Muza SR, Fulco CS, Rock PB, Cymerman A. Efficacy of residence at moderate versus low altitude on reducing acute mountain sickness in men following rapid ascent to $4300 \mathrm{~m}$. High Alt Med Biol. 2013;14:13-18.

6. Muza SR, Rock PB, Zupan MF, Miller JC, Thomas WR, Cymerman A. Residence at moderate altitude improves ventilatory response to high altitude. Aviat Space Environ Med. 2004;75:1042-1048.

7. Fulco CS, Zupan M, Muza SR, et al. Carbohydrate supplementation and endurance performance of moderate altitude residents at $4300 \mathrm{~m}$. Int J Sports Med. 2007;28: 437-443.

8. Mandolesi G, Avancini G, Bartesaghi M, Bernardi E, Pomidori L, Cogo A. Long-term monitoring of oxygen saturation at altitude can be useful in predicting the subsequent development of moderate-to-severe acute mountain sickness. Wilderness Environ Med. 2014;25: 384-391.

9. Baecke JA, Burema J, Frijters JE. A short questionnaire for the measurement of habitual physical activity in epidemiological studies. Am J Clin Nutr. 1982;36:936942.

10. Cremona G, Asnaghi R, Baderna P, et al. Pulmonary extravascular fluid accumulation in recreational climbers: a prospective study. Lancet. 2002;359:303-309. 